\title{
SmartDisability: A Smart System of Systems approach to Disability
}

\author{
Paul Whittington and Huseyin Dogan \\ Faculty of Science and Technology \\ Bournemouth University \\ Poole, United Kingdom \\ \{paul.whittington, hdogan\}@bournemouth.ac.uk
}

\begin{abstract}
This paper introduces the SmartDisability Framework; a System of Systems to consider mappings between the Disability Types, Range of Movement and Interaction Mediums to produce Technology and Task recommendations. Each element is seen as a constituent system that relies on interaction between the user and technology. The recommended technologies are viewed as independent and operable constituent systems that are networked together to assist people with disability. The SmartDisability conceptual model (based on the familiar disability symbol) and extracts from the initial development stage of the framework are presented. The framework has been populated through a systematic literature review of disability classification, Range of Movement, interaction mediums, 'off-the-shelf' technologies and tasks. The framework was augmented by the results of a previously conducted requirements elicitation process, involving surveys and semi-structured interviews, and a user evaluation with head tracking technology. Quality Function Deployment determined the relationships within the framework to ensure that user requirements were fully analysed. The anticipated validation process involving a focus group utilising fictional personas and routes to exploitation (through the development of an application) are also discussed.
\end{abstract}

Keywords-Framework; Pervasive Computing; People with Disability; Quality Function Deployment; System of Systems.

\section{INTRODUCTION}

Approximately 500 million people worldwide have disabilities that affect their interaction with society and the environment [1]. A user survey conducted by [2] highlights that people with disabilities are often unaware of how technology can provide assistance with daily tasks, thereby improving quality of life. This indicates the importance of promoting assistive technology solutions, which can be viewed from a System of Systems (SoS) perspective to develop the proposed SmartDisability Framework.

The framework has been applied to the SmartPowerchair SoS described in [3] by considering the mappings between disability type, Range of Movement (ROM), interaction mediums, technologies and tasks. It is a recommender system that uses physical impairments and ROM characteristics to suggest technologies to assist people with disability to perform daily tasks. The framework addresses the concept of not having a 'single technology solution to suit all disabilities'. It consists of seven elements; Disabilities, Impairments, Range of Movements, Movement Characteristics, Interaction Mediums, Technologies and Tasks, interlinking aspects of Human Computer Interaction. The relationship is illustrated in a linear conceptual model (Figure 1) based on the familiar disability symbol, with Disabilities being the input.

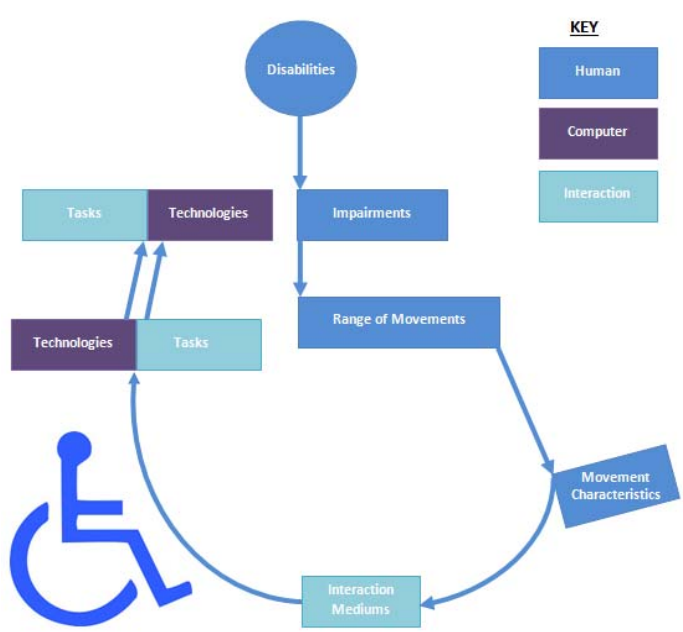

Figure 1: Conceptual model of the SmartDisability Framework

The framework is in the initial stages of development, with the seven key elements awaiting validation from domain experts and people with a variety of disabilities through focus groups and fictional personas. Involvement of people with disabilities will ensure that the key user requirements are captured by the framework. SmartDisability is in a spreadsheet format containing individual worksheets for each element with images and references provided for information purposes. The framework will be converted into a database to ensure data integrity between the elements, allowing an application to be developed for exploitation purposes.

\section{LITERATURE REVIEW}

\section{A. Disability Classification}

There are a number of disabilities that exist worldwide as human beings are susceptible to diminishing health and the possibility of developing a disability at any point during life [4]. Various frameworks have been developed to categorise 
disability, including Nagi's model in the 1950s to distribute welfare and economic aid [5], a conceptual model of the Fundamental Principles of Disability [6], the International Classification of Impairment, Disability and Handicap (ICIDH) [7] and the model by the National Center for Medical Rehabilitation Research of Bethesda's (NCMRR) for rehabilitation through adapted living environments [8].

The International Classification of Functioning, Disability and Health (ICF) Framework [9] by the World Health Organisation (WHO) is the current international standard for disability classification [10] and was a revision of the original ICIDH recognised by 191 countries [11]. The framework rationale stated that disability should not characterise individuals, but be a complex interaction method between a person and the environment [12], considering health conditions and environmental factors that result in disability. As an addition to the ICF Framework, the WHO subsequently produced the ICF-CY Framework for children and youths [13]. Research conducted to analyse the relationship between ICF, the Downton Scale and impairment types [14] has mapped disabilities into three categories; 'Motor Control', 'Senses' and 'Cognitive Ability' each with resulting impairments, such as 'increased/reduced sensation', 'weakness/paralysis/muscle wasting' and 'visual' and examples of physical disabilities, e.g. acquired brain injury, cerebral palsy and muscular dystrophy. The ICF and the classification system provided the impairment types and disabilities for the Disability element of the SmartDisability Framework.

Impairments can be classified as congenital (i.e. from birth) or acquired [15] (e.g. a traumatic injury or medical condition). Examples of congenital defects are contractures, dystonia and visual impairments. Contractures develop when normally elastic tissues are replaced by inelastic fibre-like tissues preventing normal ROM [16]. Dystonia is involuntary, variable movement noticeable when the person attempts to move [17]. Visual impairments are classed as any form of vision loss, irrespective of whether the person has partial or full loss [18]. Acquired impairments include paraplegia, cataracts and hemiparesis Paraplegia is the complete or incomplete paralysis affecting the legs and the trunk, but not the arms, as a result of a spinal cord injury where the level of injury in the spine determines the extent to which the trunk is affected [19]. Cataracts can develop at any point in life and are cloudy patches on the lens of the eye causing blurred or misty vision [20]. Hemiparesis is weakness on one side of the body where the affected side has reduced muscle strength and commonly occurs from brain haemorrhage [21].

\section{B. Range of Movement (ROM)}

ROM is a measure of movement about the axis of a joint that a person can produce using their own strength [22]. The ROM of the user was seen as a greater indicator of whether a technology would be suitable rather than a disability type due to the head tracking technology in the performed user evaluation requiring a full level of ROM. ROM is accurately measured using a goniometer between 0 to 360 degrees and for the purposes for the SmartDisability Framework, ROM was classified as a Boolean parameter, i.e. the user could or could not produce the movement. There are defined terms relating to impairments that adversely affect ROM, e.g. a limited movement of any joint is known as reduced normal Range of Motion of the joint [23], whereas, a specific impairment (e.g. finger dexterity) is the inability to manipulate objects using fingers and can be a contraindication of disabilities such as Cerebral Palsy [24]. Joint hypermobility ('double jointed') is when some or all of a person's joints have an unusually large ROM [25]. ROM can also be affected by susceptibility of joints being forced out of position, i.e. dislocation [26].

\section{Interaction Mediums}

Traditionally, interaction mediums have been touch-based, but the evolution of technology has resulted in alternative interaction methods becoming available, including monitoring brain activity [27] and gesture recognition [28]. Brain activity can be monitored using a commercially available electroencephalograph that records electrical brain activity by attaching electrodes to the scalp. A user experimentation was performed to analyse the physical and mental workload demands of touch and joystick-based interactions with a smartphone and tablet, using the iOS Switch Control built-in head tracking feature [29].

\section{Technologies}

Pervasive and assistive technologies were selected as most relevant for the Technology element of the framework as they unobtrusively support the user to interact with devices without being aware of the underlying communications. The aim of the paradigm [30] was that computers would fit into the human environment instead of humans having to enter the computer environment. Assistive technology is defined as "any product or service designed to enable independence for those with a disability or impairment" [31], which can be enhanced through the integration of pervasive technology and lead to improved usability.

\section{Methodology}

\section{A. System of Systems ( $\mathrm{SoS})$}

A SoS is defined as an "an integration of a finite number of constituent systems which are independent and operable, and which are networked together for a period of time to achieve a certain higher goal" [32], where the capability of the entire SoS is not possessed by any constituent system. SoS can be analysed using Characterisation of SoS [33] and the Capability Cube Model, initially developed for the defence industry is now applied to other domains such as transportation, healthcare, disaster response and energy. There are differences between the roles of the constituent systems and the SoS, e.g. stakeholder involvement, performance and behaviour, testing and evaluation [34].

Each element of the SmartDisability Framework is seen as a constituent system of a SoS relying on interaction between the user (the Disability, ROM and ROM Characteristics elements) and the technologies. The recommended technology 
solutions produced by the framework are viewed as constituent systems to assist users when performing tasks.

\section{B. Fictional Personas}

Fictional user personas are developed through observations and interviews of real users as suggested by [35] and will to be applied during the validation process of the SmartDisability Framework. User personas are one of four defined types [36] first introduced by [37], referred to as 'stereotyping' and are considered to be one of the most important user centred design techniques to characterise the user traits relevant for development [38]. The term 'stereotype' implies preconceived opinions; hence personas are now considered as archetypes or precise descriptions of a user and what she/he wishes to accomplish [39].

The aim of utilising personas will be for domain experts to evaluate the relationships defined by the framework through producing technology recommendations. Each persona will contain a specific type of disability, one or more physical impairments, details of ROM and the associated characteristics, and the tasks that are difficult to perform.

\section{Systematic Literature Review}

A systematic literature review was conducted to populate the SmartPowerchair Framework through establishing the relationships between the elements. This will be supplemented by knowledge from domain experts and further literature reviews. Results of surveys and interviews in the requirements elicitation phase performed by [40] provided an initial set of physical disabilities supplemented by researching other forms of disability from medical publications. The survey and semistructured interview results and observations from the participants in a head tracking evaluation enhanced the preliminary impairment types and related physical disabilities. Each impairment was associated with an affected ROM and a method of recording each type was identified through recognised physiological measuring techniques, such as extension, flexion and gaze direction. The technological elements of the framework (Interaction Mediums and Technologies) were established through analysing currentlyavailable technologies and their interaction methods from online sources. Technology user manuals and feasibility trials provided information on user interaction methods and usability. The Task element was collated from aspects of the requirements elicitation that concerned tasks that were difficult for a person with disability to perform independently in their home and therefore, required assistance from carers or parents, e.g. opening and closing doors and windows. Existing SmartHome devices, such as automated heating controls also contributed to the tasks to be supported by technology.

\section{Quality Function Deployment (QFD)}

Adopting the QFD process has allowed the SmartDisability Framework to be populated by determining the relationships between Disabilities, Impairments, ROM Characteristics and Tasks (i.e. the 'customer needs and expectations') to Interaction Mediums and Technologies (i.e. the 'technical requirements').
QFD was originally developed by [41] and is widely used in engineering industries as a structured and disciplined process providing the means to identify and carry the 'voice' of the customer through each stage of development and implementation [42]. QFD consists of a relationship matrix by converting the customer needs into technical requirements to be used in the design process. QFD was applied to intercity passenger transportation to determine the customer expectations, as illustrated by [43]. Discussions were held using focus groups to analyse existing customer requests, complaints and the distribution of a survey to current customers.

QFD ensured that the user requirements were fully analysed, enabling the framework to produce suitable technology recommendations for people with disability.

\section{RESULTS}

\section{A. Disabilities}

The Disabilities table (Figure 2) identifies the physical impairments associated with specific disability types such as an Acquired Brain Injury and Cerebral Palsy, to filter the range of disabilities into generic impairment types. The checkmarks infer that the impairment is a contraindication of a disability, colour-coded depending on the literature source. The impairment types are categorised depending on the affected body parts; 'Joints', 'Muscles', 'Vision' and 'Sensory'. The input to the table is the disability type of the user and this is used to produce a list of affected body parts, leading to input to the ROM table.

\begin{tabular}{|c|c|c|}
\hline Impairments & Acquired Brain Injury & Cerebral Palsy \\
\hline \multicolumn{3}{|l|}{ bizss } \\
\hline Limited neck movement & $\checkmark$ & $\checkmark$ \\
\hline Limited shoulder movement & $\checkmark$ & $\checkmark$ \\
\hline Limited elbov movement & $\checkmark$ & $\checkmark$ \\
\hline Limited v rist movement & $\checkmark$ & $\checkmark$ \\
\hline Limited finger dexterity & $\checkmark$ & $\checkmark$ \\
\hline Limited ankle movement & $\checkmark$ & $\checkmark$ \\
\hline \multicolumn{3}{|l|}{ Joint hypermobility } \\
\hline \multicolumn{3}{|l|}{ Joint dislocation } \\
\hline Scoliosis & & $\checkmark$ \\
\hline \multicolumn{3}{|l|}{ Mosolos } \\
\hline Contractures & $\checkmark$ & $\checkmark$ \\
\hline Dyskenesia & & $\checkmark$ \\
\hline \multicolumn{3}{|l|}{ Atrophy } \\
\hline Paraplegia & $\checkmark$ & $\checkmark$ \\
\hline Quadraplegia I tetraplegia & $\checkmark$ & $\checkmark$ \\
\hline Hemiparesis & $\checkmark$ & $\checkmark$ \\
\hline \multicolumn{3}{|l|}{ ision } \\
\hline Visual & $\checkmark$ & $\checkmark$ \\
\hline Cataracts & & \\
\hline
\end{tabular}

Figure 2: An extract of the Disabilities table

\section{B. Range of Movements (ROM)}

The Range of Movements (ROM) table (Figure 3) considers how impairment types restrict the ROM of an individual, categorised into associated ROM types. 


\begin{tabular}{|c|c|c|c|}
\hline \multirow[b]{2}{*}{ Resulting Impairments } & \multicolumn{3}{|c|}{ Associated ROM } \\
\hline & Neck & Shoulder & Elbow \\
\hline \multicolumn{4}{|l|}{ Joints } \\
\hline Limited neck movement & $\checkmark$ & & \\
\hline Limited shoulder movement & & $\checkmark$ & \\
\hline Limited elbow movement & & & $\checkmark$ \\
\hline \multicolumn{4}{|l|}{ Limited wrist movement } \\
\hline \multicolumn{4}{|l|}{ Limited finger dexterity } \\
\hline \multicolumn{4}{|l|}{ Limited ankle movement } \\
\hline Joint hypermobility & & $\checkmark$ & $\checkmark$ \\
\hline Joint dislocation & $\checkmark$ & $\checkmark$ & $\checkmark$ \\
\hline Twisted spine & $\checkmark$ & $\checkmark$ & \\
\hline \multicolumn{4}{|l|}{ Muscles } \\
\hline Contractures & $\checkmark$ & $\checkmark$ & $\checkmark$ \\
\hline Dyskenesia & $\checkmark$ & $\checkmark$ & $\checkmark$ \\
\hline Atrophy & $\checkmark$ & $\checkmark$ & $\checkmark$ \\
\hline Paraplegia & & & \\
\hline
\end{tabular}

Figure 3: An extract of the ROM table

The ROM table forms an input to the ROM Characteristics table, where depending on which type of ROM is affected by the individuals' impairment, the appropriate information is obtained.

\section{ROM Charactistics}

The measurable features of each ROM type are identified in the ROM Characteristics table (Figure 4) and have a number of characteristics that determine how the ROM is affected by the impairments. The table uses Boolean statements to determine whether the user can perform each movement and is the input to the Interaction Mediums table, enabling suitable interaction mediums to be recommended.

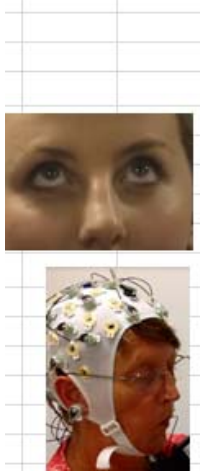

\begin{tabular}{|c|c|c|c|}
\hline \multirow[t]{2}{*}{ ROM Characteristic } & \multicolumn{3}{|c|}{ Measurement Type } \\
\hline & Max Degrees & Boolean & Max Percentage \\
\hline \multicolumn{4}{|l|}{ Eye ${ }^{[2]}$} \\
\hline Gaze up & & $\mathrm{Y} / \mathrm{N}$ & \\
\hline Gaze down & & $\mathrm{Y} / \mathrm{N}$ & \\
\hline Gaze left & & $\mathrm{Y} / \mathrm{N}$ & \\
\hline Gaze right & & $\mathrm{Y} / \mathrm{N}$ & \\
\hline Blinking & & $\mathrm{Y} / \mathrm{N}$ & \\
\hline \multicolumn{4}{|l|}{ Mouth ${ }^{[4]}$} \\
\hline Suck & & $\mathrm{Y} / \mathrm{N}$ & \\
\hline Blow & & $\mathrm{Y} / \mathrm{N}$ & \\
\hline Bite tongue & & $\mathrm{Y} / \mathrm{N}$ & \\
\hline Move tongue left & & $\mathrm{Y} / \mathrm{N}$ & \\
\hline Move tongue right & & $\mathrm{Y} / \mathrm{N}$ & \\
\hline Smiling & & $\mathrm{Y} / \mathrm{N}$ & \\
\hline
\end{tabular}

Figure 4: An extract of the ROM Characteristics table

\section{Interaction Mediums}

Figure 5 describes the relationship between different interaction mediums and the required ROM for the interaction between a user and technology. The cells of the table are highlighted where the interaction medium requires a particular ROM characteristic, e.g. an eye-based medium requires a user to gaze up, down, left, right or blink. 'Sip n Puff' is an assistive technology device that requires a user to interact through sucking and blowing [44]. Gesture control enables users to create specific gestures with their hands to interact with devices [28], but is only suitable for users who have full elbow, wrist and hand ROM. The output of the table is a list of interaction mediums that are suitable for the user and represents the input to the Technologies table with any mediums that require a ROM that the user does not possess, are omitted from the recommendation.

\begin{tabular}{|l|l|l|l|l|l|}
\hline \multicolumn{1}{|c|}{$\begin{array}{c}\text { Associated } \\
\text { Characteristic }\end{array}$} & \multicolumn{5}{c|}{ Interaction Mediums } \\
\hline & $\begin{array}{l}\text { Joystick } \\
{[1]}\end{array}$ & $\begin{array}{l}\text { Voice } \\
{\left[{ }^{6} \text { Eye }\right.}\end{array}$ & Head $^{\left[{ }^{[3]}\right.}$ & Eye ${ }^{\left[{ }^{[2]}\right.}$ & $\begin{array}{l}\text { Sip n Puff } \\
{\left[{ }^{[4]}\right.}\end{array}$ \\
\hline Gaze up & & & & & \\
\hline Gaze down & & & & & \\
\hline Gaze left & & & & & \\
\hline Gaze right & & & & & \\
\hline Blinking & & & & & \\
\hline \multicolumn{1}{|c|}{ Mouth } & & & & & \\
\hline Suck & & & & & \\
\hline Blow & & & & & \\
\hline Bite tongue & & & & & \\
\hline Move tongue left & & & & & \\
\hline Move tongue right & & & & & \\
\hline Smiling & & & & & \\
\hline & & & & & \\
\hline Speech intelligability & & & & & \\
\hline
\end{tabular}

Figure 5: An extract of the Interaction Mediums table

\section{E. Technologies}

The Technologies table (Figure 6) identifies the specific technologies that can be operated through each interaction medium, such as smartphones, tablets and built-in eye tracking.

\begin{tabular}{|l|l|l|l|l}
\hline \multirow{2}{*}{$\begin{array}{c}\text { Interaction } \\
\text { Mediums }\end{array}$} & \multicolumn{5}{c}{ Technology } \\
\cline { 2 - 5 } & $\begin{array}{c}\text { Smartphone } \\
{[1]}\end{array}$ & Tablet [2] & $\begin{array}{c}\text { Head Mounted } \\
\text { Display [3] }\end{array}$ & $\begin{array}{c}\text { Built-in Eye Tracking } \\
{[4]}\end{array}$ \\
\hline Joystick & & & & \\
\hline Voice & & & & \\
\hline Head & & & & \\
\hline Eye & & & & \\
\hline Sip n Puff & & & & \\
\hline Foot & & & & \\
\hline Chin & & & & \\
\hline Fingers & & & & \\
\hline Brain activity & & & & \\
\hline
\end{tabular}

Figure 6: An extract of the Technologies table

A head mounted display and an electroencephalogram are classed as wearable, as the user is required to wear the technology to interact with it. The feasibility trials illustrated that there was an obvious limitation if a person did not possess the required dexterity. Eye tracking can either be a built-in feature of a device (e.g. smartphone) or stand-alone, which specifically captures the eye movements of the user. Momentary switches enable the user to interact with devices by pressing buttons located in any position, e.g. the headrest or arm of a powerchair. A rear view camera would assist the user with manoeuvring from a live view on a smartphone or tablet attached to the powerchair. Most of the technologies have multiple methods of interaction, e.g. smartphones can be used by fingers, joystick, head, eye, 'Sip n Puff' and voice, whereas built-in head tracking can only be used with the head. The head tracking experimentation proved that iOS Switch Control was only suitable for users who possessed the necessary neck ROM. The table provides technology recommendations that are suitable for the ROM of 
the users and will be the input to the final table of the SmartDisability Framework; the Tasks table.

\section{F. Tasks}

The purpose of the Tasks table (Figure 7) is to suggest daily tasks that can be performed with each of the technologies defined in the Technologies table. Most of the technologies can support a variety of tasks whereas, some are specific e.g. a rear view camera can only assist with navigation. The output of the table produces a list of recommended technologies and supported tasks that are suitable for the disability of the user.

\begin{tabular}{c|c|l|l|}
\hline \multirow{2}{*}{ Technology } & \multicolumn{3}{c|}{ Task } \\
\cline { 2 - 4 } & $\begin{array}{l}\text { Navigating } \\
\text { powerchair } \\
{[1]}\end{array}$ & $\begin{array}{l}\text { Operating vehicle } \\
\text { adaptations [1] }\end{array}$ & $\begin{array}{l}\text { Operating cooking } \\
\text { equipment [1][2] }\end{array}$ \\
\hline Smartphone & & & \\
\hline Tablet & & & \\
\hline Head Mounted Display & & & \\
\hline Built-in Eye Tracker & & & \\
\hline Stand-alone Eye Tracker & & & \\
\hline Electroencephalogram & & & \\
\hline Momentary Switches & & & \\
\hline Rear View Camera & & & \\
\hline
\end{tabular}

\section{Figure 7: An extract of the Tasks table}

\section{Discussions}

The aim of the SmartDisability Framework was to recommend interaction mediums, technologies and tasks depending on the disability, impairments and ROM of the user, thereby avoiding a 'single solution to fit all'. The conceptual model of the SmartDisability Framework (Figure 1) illustrated a SOS relies on the interactions and interoperability between technologies and the user. The technologies included in the framework are viewed as independent and operable constituent systems that are networked together for a period of time [32] to assist a person with disability and provide task interaction.

Analysis of a range of physical disabilities identified common impairments used to characterise the types of ROM that are affected by disability. The impairment types were classified and formed the basis of the ROM element of the framework. Measurement techniques were applied to the ROM Characteristics element resulting in Boolean parameters. The characteristics were used to determine the suitable interaction mediums, as each medium was related to a required ROM. Currently available technologies were contained within the Technology element and each had defined supported interaction mediums. The Task element described daily tasks that users wished to perform with the assistance of technology. The relationships between technologies and tasks were established from tasks that were difficult for people with disabilities to perform to investigate whether new technologies could provide an alternative method of performing a task.

The framework will be validated through the involvement of experts from the domains of healthcare, computing and occupational therapy, with the healthcare domain validating the disabilities, impairments and ROM sections, computing validating the technology related aspects and occupational therapists validating the tasks. The validation process will take the form of a focus group where each participant will be allocated at least two disabilities, ROM, ROM Characteristics, Technologies or Tasks. Fictional personas will also be applied to test the framework consisting of the physical impairments and the ROM of a fictional individual and asked to create technology recommendations for a persona. The results will be validated to see whether the technology and tasks will be applicable to an individual. A concluding open discussion will be held to discuss all aspects of the framework and a further validation exercise will be scheduled if required.

The validated framework will be disseminated to assistive technology industries, as well as to healthcare professionals to suggest technologies that could support the daily lives of their clients with disability. It is anticipated that the framework will be expanded in the future through the creation of additional columns and rows in the tables, as new forms of interaction methods, technologies and tasks are established. To maintain the framework integrity, it will be necessary for any new aspect to be mapped to an associated element.

\section{CONCLUSIONS}

The proposed SmartDisability Framework is a SoS that recommends technology solutions depending of the impairments and ROM of the user. It relies on the interaction of constituent, 'off-the-shelf' technologies through a variety of mediums to assist people with disability to perform daily tasks that would otherwise be challenging. The initial development phase of the framework was the result of a systematic literature review into disability classification and impairments, ROM types and characteristics, technologies and tasks. This was supplemented by the findings and observations of a previously conducted requirements elicitation study into the difficulties that people with disability encounter and a head tracking user experimentation. The framework considered the wide-range of possible physical disabilities and condensed them into a set of resulting impairments that were used to characterise the affected ROM of a user.

The initial framework is the first stage of the research that is to be validated, with expected dissemination and exploitation through a smartphone or web-based application. Users would input their impairments, and associated ROM Characteristics to the application and by utilising the contents of the framework, technology recommendations with supported tasks would be suggested. The application will be used by either people with disability to discover technologies that are available, or by industries and healthcare professionals to assist their clients with disability in their daily lives. Considering the proliferation of smart technology over recent years [45], it is anticipated that by applying SoS to disability through the SmartDisability Framework, disability can become 'Smart' to potentially improve quality of life and provide independence. 


\section{REFERENCES}

[1] J. P. Cofré et al., "Developing a Touchscreen-based Domotic Tool for Users with Motor Disabilities" in 2012 Ninth International Conference on Information Technology: New Generations, Las Vegas, NV, 2013, pp. 696-701.

[2] I. A. Ari and F. A. Inan, 2010. "Assistive Technologies for Students with Disabilities: A Survey of Access and Use in Turkish Universities", Turkish Online J. Educational Technology, vol. 9, no. 2, pp 40-45, Jan. 2010.

[3] P. Whittington and H. Dogan, "SmartPowerchair: A Pervasive System of Systems" in 10th System of Systems Engineering Conference, San Antonio, TX, USA, 2015, pp. 244-249.

[4] N. Kostanjsek, 2011. "Use of The International Classification of Functioning, Disability and Health (ICF) as a conceptual framework and common language for disability statistics and health information systems", BMC Public Health, vol. 11, no. 4, pp 1-6, May 2011.

[5] S. Z. Nagi, 1965. "Some conceptual issues in disability and rehabilitation", Sociology and Rehabilitation, Jan. 1965.

[6] Union of the Physically Impaired Against Segregation, Fundamental Principles of Disability. London, UK, UPIAS, 1976.

[7] World Health Organization, International Classification of Impairment, Disability and Handicap (ICIDH). Geneva, Switzerland, WHO, 1980.

[8] National Center for Medical Rehabilitation Research. Report to the NACHHD Council. Washington, WA, USA: US Department of Health and Human Services, 2006.

[9] World Health Organization, International Classification of Functioning, Disability and Health (ICF) Framework. Geneva, Switzerland, WHO, 2001.

[10] R. E. Cowan et al., 2012. "Recent trends in assistive technology for mobility", J. NeuroEngineering and Rehabilitation, vol. 9, no. 20, pp. 18. Apr. 2012

[11] C. Masala and D. R. Petretto, 2008. "From disablement to enablement: Conceptual models of disability in the 20th century", Disability and Rehabilitation, vol. 30, no. 17, pp. 1233-1244, Jul. 2009.

[12] N. Kostanjsek, 2011. "Use of The International Classification of Functioning, Disability and Health (ICF) as a conceptual framework and common language for disability statistics and health information systems", BMC Public Health, vol. 1, no. 4, pp. 1-6, May 2011.

[13] World Health Organization, ICF-CY Framework for children and youths. Geneva, Switzerland, WHO, 2007.

[14] R. Andrews, "Disabling conditions and ICF measures", unpublished.

[15] University of Wisconsin-Extension, (2010, Apr.). "Coping with a Disability" [Online]. Available: fyi.uwex.edu/agrability/files/2010/04/4Coping-with-a-Disability.ppt

[16] U.S. National Library of Medicine, "Contracture deformity" [Online]. Available: https:/www.nlm.nih.gov/medlineplus/ency/article/003185.htm

[17] Cerebral Palsy Alliance, "Dyskinetic Cerebral Palsy" [Online]. Available: $\quad$ https://www.cerebralpalsy.org.au/what-is-cerebralpalsy/types-of-cerebral-palsy/dyskinetic-cerebral-palsy.

[18] The Nemours Foundation, "Visual Impairment" [Online] Available: http://kidshealth.org/teen/diseases_conditions/sight/visual_impairment.h tml.

[19] Spinal Injury Network, "What is Paraplegia?" [Online] Available: http://www.spinal-injury.net/paraplegia.htm

[20] Department of Health, "Age-related cataracts" [Online]. Available: http://www.nhs.uk/conditions/Cataracts-agerelated/Pages/Introduction.aspx

[21] National Stroke Association, "Hemiparesis" [Online] Available: http://www.stroke.org/we-can-help/survivors/stroke-recovery/poststroke-conditions/physical/hemiparesis

[22] G. Keilhofner, Research in Occcupational Therapy: Methods of Inquiry for Enhancing Practice. Philadelphia, PA: F.A. Davis Company, 2006, pp. 157.

[23] K. O'Connell, (2012, Sep. 17). "What causes limited range of motion? 20 possible conditions" [Online]. Available: http://www.healthline.com/symptom/limited-range-of-motion
[24] Georgia Tech Research Institute, (2007). "Dexterity and Mobility Impairment Fact Sheet" [Online]. Available: http://accessibility.gtri.gatech.edu/assistant/acc_info/factsheet_dexterity _mobility.php

[25] Department of Health, "Joint Hypermobility" [Online]. Available: http://www.nhs.uk/conditions/Jointhypermobility/Pages/Introduction.aspx

[26] U.S. National Library of Medicine, "Dislocations" [Online]. Available: https://www.nlm.nih.gov/medlineplus/dislocations.html

[27] United News Network GmbH., (2010, Apr. 14). "Official patent approval for the "actiCAP" EEG cap approaches" [Online]. Available: http://www.pressebox.com/pressrelease/brain-products/Official-patentapproval-for-the-actiCAP-EEG-cap-approaches/boxid/336978

[28] J. Platz and K. Clothier, (2015, Aug. 19). "Motion-controlled Servos with Leap Motion \& Raspberry Pi" [Online]. Available: https://www.pubnub.com/blog/2015-08-19-motion-controlled-servoswith-leap-motion-raspberry-pi/

[29] Komodo OpenLab Inc., (2014, Jun 05). "iPhone and iPad accessibility options for quadriplegics and other users with limited mobility" [Online]. Available: http://gettecla.com/blogs/news/14347593-iphoneand-ipad-accessibility-options-for-quadriplegics-and-other-users-withlimited-mobility

[30] M. Weiser, 1991. "The Computer for the 21st Century". Scientific American, vol. 265, no. 3, pp. 66-75, Sep. 1991.

[31] R. Andrews, (2015, Jul.). "Latest Developments in Assistive Technology". Cambridge University Hospitals, Cambridge, UK: [Online]. Available: http://www.erbifinfo.org/media/1923/ERBIFPresentation-July-2015.pdf.

[32] M. Jamshidi. Systems of Systems Engineering - Innovations for the 21st Century. Hoboken, NJ, USA: John Wiley \& Sons, 2009.

[33] A. Harding et al., "A structured approach to planning and managing systems engineering capability evolution in a complex through-life business space" in 7th Annu. Conf. Sys. Eng. Research, Loughborough, UK, 2009, pp. 3-5.

[34] V. Kinder et al., "System of Systems: defining the System of Interest" in 7th Int. Conf. System of Syst. Eng, Genoa, Italy, 2012, pp. 463-468.

[35] W. Quesenbery. (2003, Jun 18). "Personas: Bringing Users Alive" [Online]. Available: http://www.wqusability.com/handouts/personasoverview.pdf

[36] A. Madureira et al. "Using Personas for Supporting User Modeling on Scheduling Systems" in 14th International Conference on Hybrid Intelligent Systems, Kuwait, 2014, pp. 279-284.

[37] A. Cooper. The Inmates Are Running the Asylum: Why High Tech Products Drive Us Crazy and How to Restore the Sanity. Indianapolis, IN, USA: Sams Publishing, 2004.

[38] P. T. Aquino Junior and L. V. L. Filgueiras, "User modeling with personas" in 2005 Latin American conference on Human-Computer Interaction, Cuernavaca, Mexico, 2005, pp. 277-282.

[39] D. Hix and H. R. Hartson. Developing user interfaces: Ensuring usability through product and process, New York, NY, USA: John Wiley \& Sons, 1993.

[40] P. Whittington et al., "SmartPowerchair: To boldly go where a powerchair has not gone before" in Ergonmics \& Human Factors 2015, Daventry, UK, 2015, pp. 233-240.

[41] Y. Akao. Quality Function Deployment. Cambridge, MA, USA: Productivity Press, 1990.

[42] J. B. Re Velle et al. The QFD Handbook. New York, NY, USA: John Wiley \& Sons, 1998.

[43] F. Pakdil and F. B. Kurtulmuşoğlu, "Improving service quality in highway passenger transportation: a case study using quality function deployment”. Eur. J. Transport and Infrastructure Res., vol. 14, no. 4, pp. 375-393, Apr. 2014.

[44] Origin Instruments Corporation, (2015). "Sip and Puff Switch Solutions: Single and Multi-User Offerings" [Online]. Available: http://www.orin.com/access/sip_puff/

[45] G. Suarez-Tangil et al., 2013. "Evolution, Detection and Analysis of Malware for Smart Devices", Communications Surveys \& Tutorials, vol.16, no.2, pp. 961- 987, Apr. 2014. 